\title{
Complete Response of Isolated Para-aortic Lymph Node Recurrence from Rectosigmoid Cancer Treated by Chemoradiation Therapy with Capecitabine/Oxaliplatin plus Bevacizumab: A Case Report
}

\author{
Tomonori Miyazawa $^{a} \quad K^{2} a z u y u E^{b} e^{b}$ Norihiko Koide $^{a}$ \\ Nobuhiro Fujita ${ }^{a}$ \\ Departments of a Surgery and ${ }^{b}$ Radiation Therapy, Joetsu General Hospital, \\ Joetsu, Japan
}

\section{Key Words}

Chemoradiation therapy - Para-aortic lymph node recurrence - Colorectal cancer . Capecitabine/oxaliplatin plus bevacizumab

\begin{abstract}
Para-aortic lymph node recurrence is a rare type of metastasis from colorectal cancer, and no treatment has yet been established. Here, we report on a case of isolated para-aortic lymph node metastasis from rectosigmoid cancer that showed complete response to chemoradiation therapy with capecitabine/oxaliplatin plus bevacizumab. A 58-year-old woman underwent high anterior resection for rectosigmoid cancer in 2009. Para-aortic lymph node recurrence occurred in 2011. She underwent radiation therapy (50 Gy) and 8 courses of capecitabine/oxaliplatin plus bevacizumab. Abdominal computed tomography and positron emission tomography with 18-fluorodeoxyglucose did not reveal any paraaortic lymph node recurrence after chemoradiation therapy. Hence, this case was interpreted as a complete response. No recurrence was noted 6 months after the complete response. Chemoradiation therapy with capecitabine/oxaliplatin plus bevacizumab is likely to be effective in treating patients with para-aortic lymph node recurrence.
\end{abstract}




\section{Introduction}

Since the appearance of new anti-cancer drugs and molecular target agents, chemotherapeutic treatment of metastatic colorectal cancer (MCRC) has enhanced the quality of life for patients and has improved both progression-free survival (PFS) and overall survival (OS) [1]. Capecitabine/oxaliplatin (XELOX) is non-inferior to fluorouracil/folinic acid plus oxaliplatin (FOLFOX-4) as first-line chemotherapy for patients with MCRC [2]. The addition of bevacizumab to FOLFOX-4 or XELOX significantly improved PFS in the first-line treatment of MCRC [3].

Para-aortic lymph node recurrence (PALR) is a rare type of MCRC, and no treatment has yet been established $[4,5]$. Here, we report on a case of PALR from rectosigmoid cancer that showed complete response (CR) to chemoradiation therapy with XELOX plus bevacizumab.

\section{Case Report}

A 58-year-old woman underwent high anterior resection for rectosigmoid cancer in 2009. Pathological examination showed a moderately differentiated adenocarcinoma perforating the visceral peritoneum, with metastatic involvement in 3 of 12 lymph nodes removed (T4 N1). She underwent 5 cycles of oral tegafur/uracil plus leucovorin therapy as postoperative adjuvant chemotherapy. In 2011, an abdominal computed tomography (CT) scan showed swollen para-aortic lymph nodes ( $\underline{\text { fig. 1a }}$, b). Positron emission tomography with 18-fluorodeoxyglucose (FDG-PET)/CT fusion imaging indicated FDG accumulations at the right side of the abdominal aorta (fig. 1c). Thus, the patient was diagnosed with PALR from rectosigmoid cancer. In May 2011, we started para-aortic radiation of 50 Gy in 25 fractions and XELOX plus bevacizumab [oxaliplatin $\left(130 \mathrm{mg} / \mathrm{m}^{2}\right)$ and bevacizumab $(7.5 \mathrm{mg} / \mathrm{kg})$ on day 1 plus capecitabine $\left(1,000 \mathrm{mg} / \mathrm{m}^{2}\right)$ twice on days $1-14$, every 3 weeks]. After the third course of chemotherapy, the patient experienced G3 neutropenia, and the subsequent chemotherapy doses were reduced: capecitabine was reduced by 1 dose level and oxaliplatin was reduced to $100 \mathrm{mg} / \mathrm{m}^{2}$. Meanwhile, no dose modification of bevacizumab was performed.

An abdominal CT scan and a FDG-PET/CT scan were performed after the radiation therapy and 8

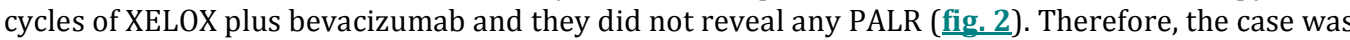
considered to be a CR. As adjuvant therapy after radiation and XELOX plus bevacizumab, 8 cycles of capecitabine [900 mg/body, twice on days 1-14, every 3 weeks] were administered. No sign of recurrence or metastasis was noted 6 months after CR was confirmed.

\section{Discussion}

The introduction of oxaliplatin and irinotecan into combination regimens with fluorouracil (5-FU), as well as the development of molecular target agents, has been a major advance in the treatment of patients with MCRC. Capecitabine exerts its anticancer effects by generating 5 -FU, preferentially through upregulation of thymidine phosphorylase (TP) in tumor cells relative to normal cells. Capecitabine generates a significantly higher response rate and displays a superior safety profile compared with bolus 5-FU/leucovorin [1]. The N016966 study demonstrated that XELOX was noninferior to FOLFOX-4 in terms of PFS, OS, and overall response rate, and the addition of bevacizumab to XELOX or FOLFOX-4 significantly improved PFS in the first-line treatment of MCRC $[3,4]$. The same study showed a longer median OS in the XELOX plus bevacizumab arm compared with the XELOX plus placebo arm (21.4 vs. 19.2 
months, respectively) [4]. The overall rate of grade $3 / 4$ adverse events was similar for FOLFOX-4 and XELOX, and the addition of bevacizumab did not alter similarities or differences between the safety profile of FOLFOX-4 and XELOX [3]. Since capecitabine is given orally and oxaliplatin and bevacizumab are administered every 21 days, XELOX plus bevacizumab reduces the need for intravenous drug administration and associated visits to the clinic. XELOX plus bevacizumab can be considered as a standard first-line treatment for patients with MCRC.

The common sites of metastasis from colorectal cancer (CRC) are the liver and lung. Complete resection of hepatic or lung metastasis results in prolonged survival and constitutes a cure in well-selected patients [6]. However, isolated PALR from CRC is rarely encountered. Min et al. [4] reported the incidence of PALR to be 1.3\% in 2,916 patients who underwent curative surgery for CRC. Therefore, the treatment for isolated PALR from CRC has not been well established. The median survival time after PALR was 34 months for the patients who underwent surgical resection and 13 months for those who underwent radiation therapy (32.4-50.4 Gy) with concurrent or sequential chemotherapy or only systemic chemotherapy $(p=0.034)$ [4]. Although most of the patients who were treated in the early 1990s might not receive the benefits of the recent developments in chemoradiation treatments, curative resection of PALR might achieve long-term survival in selected cases [4]. Kim et al. [5] reported the efficacy of stereotactic radiation to treat PALR from CRC. Seven patients with isolated PALR from rectal cancer were treated with stereotactic radiation of 36-51 Gy in 3 fractions. The study demonstrated that the 1 - and 3-year survival rates were 100 and $71.4 \%$, respectively, and the median survival time was 37 months. Fujii et al. [7] reported on a case where PALR showed CR to oral tegafur/uracil therapy in elderly rectal cancer patients.

Preoperative chemoradiation using bevacizumab, capecitabine, oxaliplatin, and standard doses of radiation for patients with high-risk rectal cancer achieved a high pathological CR rate [8]. Radiation enhances the expression of TP in tumor tissue [9]. The upregulation of TP by radiation may increase preferential delivery of 5-FU to the tumor following the administration of capecitabine.

In conclusion, we report on a case of rectosigmoid cancer with PALR that showed CR to chemoradiation therapy with XELOX plus bevacizumab. Chemoradiation therapy with XELOX plus bevacizumab is likely to be effective in treating patients with PALR.

\section{Disclosure Statement}

The authors have no conflicts of interest to declare. 

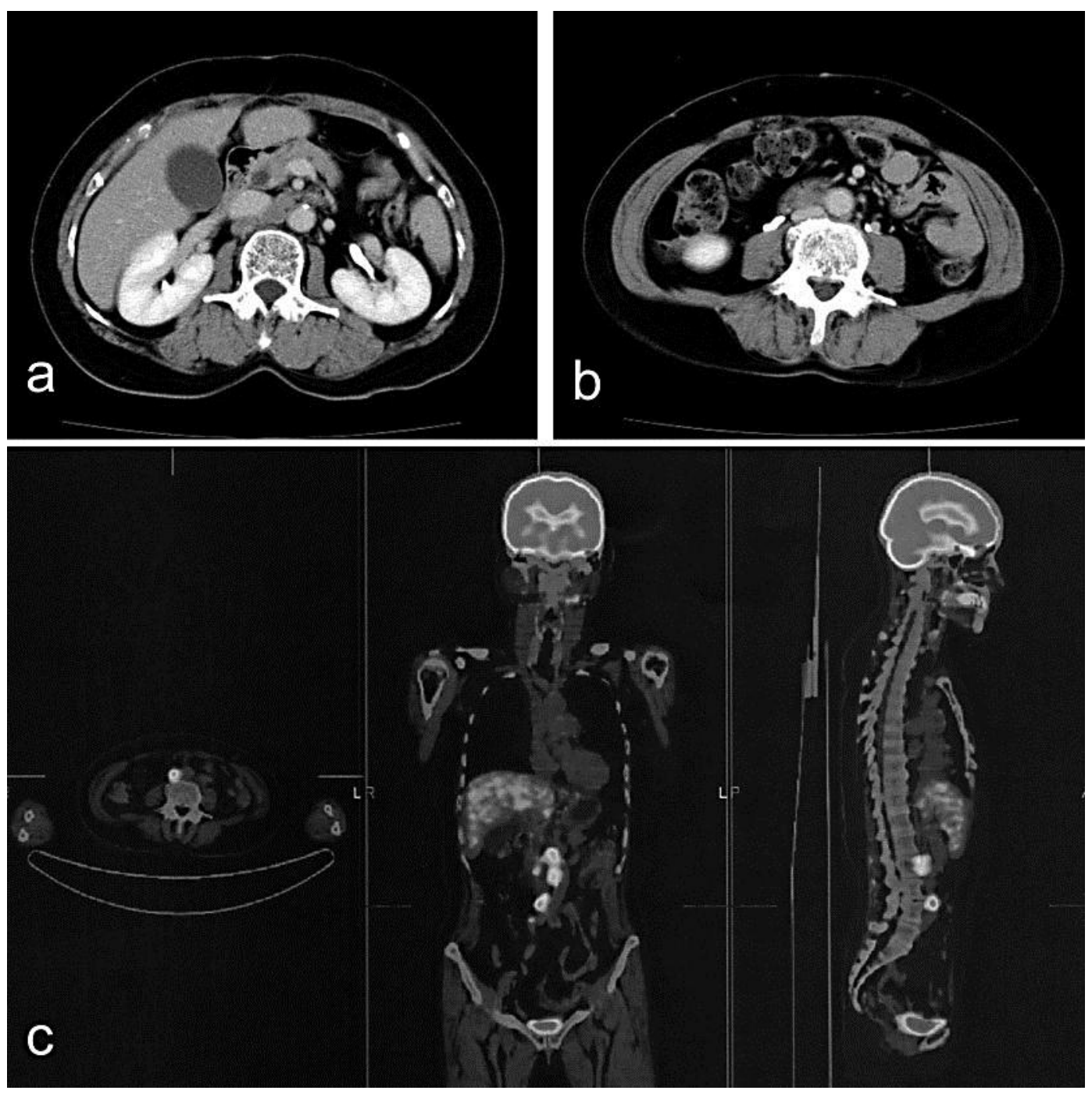

Fig. 1. Imaging before chemoradiation therapy. Abdominal CT scan showed para-aortic lymph node swelling $(\mathbf{a}, \mathbf{b})$. FDG-PET indicated FDG accumulations at the right side of the abdominal aorta (c). 

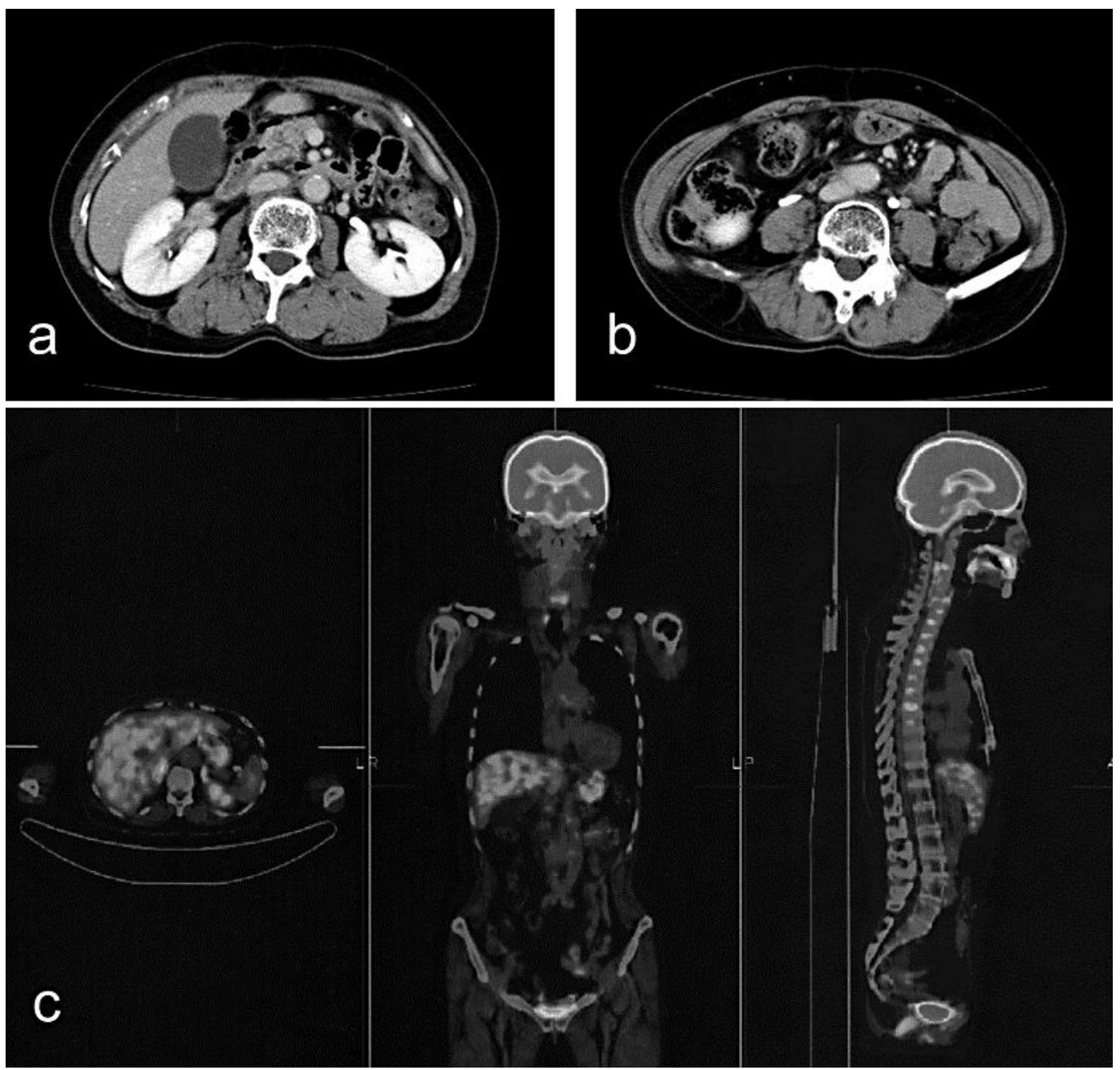

Fig. 2. Imaging after chemoradiation therapy. Abdominal CT scan (a, b) and FDG-PET (c) did not reveal any PALR.

\section{References}

1 Braum AH, Achterrath W, Wilke H, Vanhoefer U, Harstrick A, Preusser P: New systemic frontline treatment for metastatic colorectal carcinoma. Cancer 2004;100:1558-1577.

2 Cassidy J, Claeke S, Diaz-Rubio E, Scheithauer W, Figer A, Wong R, Koski S, Lichinitser M, Yang TS, Rivera F, Couture F, Sizen F, Saltz L: Randomized phase III study of capecitabine plus oxaliplatin compared with fluorouracil/folinic acid plus oxaliplatin as first-line therapy for metastatic colorectal cancer. J Clin Oncol 2008;26:2006-2012.

-3 Saltz LB, Clarke S, Diaz-Rubio E, Scheithauer W, Figer A, Wong R, Koski S, Lichinitser M, Yang TS, Rivera F, Couture F, Sizen F, Cassidy J: Bevacizumab in combination with oxaliplatin-based chemotherapy as first-line therapy in metastatic colorectal cancer: a randomized phase III study. J Clin Oncol 2008;26:2013-2019.

-4 Min BS, Kim NK, Sohn SK, Cho CH, Lee KY, Baik SH: Isolated paraaortic lymph-node recurrence after the curative resection of colorectal carcinoma. J Surg Oncol 2008;97:136-140.

5 Kim SK, Cho CK, Yang KM, Lee DH, Moon SM, Shin YJ: Stereotactic body radiotherapy for isolated paraaortic lymph node recurrence from colorectal cancer. World J Gastroenterol 2009;15:6091-6095. 
6 Mahmoud N, Dunn KB: Metastasectomy for stage IV colorectal cancer. Dis Colon Rectum 2010;53:10801092.

7 Fujii S, Ota M, Ichikawa Y, Yamagishi S, Osada S, Suwa H, Kunisaki C, Ohki S, Endo I: Paraaortic lymph node metastasis showed CR to UFT/LV therapy in elderly rectal cancer. Hepat Gastroentorology 2010;57:472-476.

8 Kennecke H, Berry S, Wong R, Zhou C, Tankel K, Easaw J, Rao S, Post J, Hay J: Pre-operative bevacizumab, capecitabine, oxaliplatin and radiation among patients with locally advanced or low rectal cancer: a phase II trial. Eur J Cancer 2012;48:37-45.

9 Glynne-Jones R, Dunst J, Sebag-Montefiore D: The integration of oral capecitabine into chemoradiation regimens for locally advanced rectal cancer: how successful have we been? Annal Oncol 2006;17:361371. 